\title{
On the Impact of the Sensing Rate of Secondary Users in Cognitive Radio Networks
}

\author{
O.I. Salameh, K. De Turck, S. Wittevrongel, S. De Vuyst, H. Bruneel \\ SMACS Research Group, \\ Ghent University, Dept. of Telecommunications and Information Processing, \\ St.-Pietersnieuwstraat 41, B-9000 Gent, Belgium. \\ Phone: +32-9-2648902, Fax: +32-9-2644295 \\ Email: $\{$ osalameh, kdeturck, sw, sdv, hb\}atelin.ugent. be
}

\begin{abstract}
The first phase in the operation of a Cognitive Radio Network (CRN) is the investigation of the availability of idle channels through channel sensing. In this paper we study the impact of the sensing rate of Secondary Users (SUs) in a CRN. We develop a continuous-time Markov chain (CTMC) to study the effect of the sensing rate $\sigma$ on the performance measures of the CRN including the expected SU delay, the SU interruption probability, the probability a SU is discarded after entering the system and the SU blocking probability. The obtained results reveal that the sensing rate has a crucial impact on these measures.
\end{abstract}

Index Terms-cognitive radio; performance evaluation; continuous-time Markov chain.

\section{INTRODUCTION}

The cognitive radio network (CRN) paradigm for wireless communications [1] is a radical approach to the spectrum shortage crisis that affects modern wireless communication networks. This spectrum shortage is due to the marked rise in popularity of wireless communications on the one hand, and the heavy underutilization of most frequency bands in the radio spectrum (often below 10\%, as shown in a.o. [2], [3]) on the other.

In wireless telecommunication, interference between different transmitters is done away with by means of band-limited radio technologies, such that only a small part of the usable radio spectrum (a frequency band or channel) is affected by the transmission and such that co-located transmissions on nonoverlapping bands do not interfere with each other. Regulatory bodies are responsible for defining which spectrum bands can be used by whom and by which technology.

The CRN paradigm originates from the observation that although most of the frequency bands in the radio spectrum are licensed and reserved, they are mostly heavily underutilized. To counter this, the central idea of $\mathrm{CRN}$ is to provide room in the licensed spectrum bands so as to accommodate secondary (unlicensed) users (SU) without disrupting the communications of the primary (licensed) users (PU). For this to work, the SU mobile devices need to be equipped with radio interfaces that have cognitive capabilities, i.e. they need to be able to detect spectrum usage in the environment (which is called sensing) and intelligently adapt their transmitting behavior 978-1-4799-0059-6/13/\$31.00 @ 2013 IEEE accordingly (action). These cognitive abilities are together known as the cognitive cycle. In this paper, we concentrate on the effect of sensing (and in particular, of the sensing rate) on the performance of SUs. We clarify the contributions in the remaining paragraphs of this section by offsetting them with prior related work

Several approaches have been used to investigate the performance of SUs in a CRN. In [4] a Markovian multiserver queueing system with preemptive priority and $r$ kinds of user classes is presented. The main performance measures considered are the expectation and the variance of the life time for a customer of class $p$. A limitation of this work is that the authors assume that a high-priority customer can interrupt a low-priority customer randomly only when all servers are busy, which seems to run counter to the spirit of CRN, i.e. PUs are assumed to be oblivious to the actions of SUs. In [5] a prioritized Markovian approach is suggested for dynamic spectrum access. Continuous-time Markov chains (CTMCs) with and without queueing that model the interaction between PUs and SUs are developed. These CTMC models are used to derive optimal access probabilities of SUs. This work considers the case that several SUs can transmit simultaneously on the same band at the same time without collisions. A limitation of this work is that it assumes that a SU does not change its channel after interruption. In [6] a loss model with a finite user population is presented. The impact of the PU load and spectrum band allocation on the delay performance of SUs is investigated based on a CTMC. All these models do not take the sensing ability of SUs into consideration.

Other research studies are based on an ON/OFF model to describe channel availability [7]-[9]. The ON state represents a busy channel by a PU and the OFF state represents an idle one. The idle state is regarded as a spectrum opportunity for SUs. These models are slot-based and it is assumed that a SU senses the spectrum and decides on its target channel at the beginning of each time slot. This assumption may lead to SU channel evacuation in the next time slot even if no PU appears on the current channel [10]. Priority M/G/1 models are also proposed to analyze CRN performance [11], [12]. As in [5], these models assume that an interrupted SU must stay at the current channel for retransmission. 
Some recent publications [13], [14] have incorporated the sensing time explicitly into their analytical models to assess the performance of SUs, but this was in the context of SU hand off scenarios. These papers provide different definitions of the sensing time. In [13] the authors define the sensing delay as the time from the moment a collision happens between a PU and a SU until the collision is detected by the SU. In [14] the term sensing time refers to the time period until a SU finds an idle channel after collision. Both these definitions are collision related and do not take into consideration the SU's need to sense while there are no collisions.

In this paper, we define the sensing time as the time a SU needs to scan the spectrum and to identify the idle and busy channels. This activity happens every time a SU attempts to access the network, regardless of previous attempts/transmissions if any. We furthermore assume that a SU vacates instantly the occupied channel in case of a PU arrival as in [15], i.e. the overlap of the SU and the PU is negligible. We propose a CTMC and derive various performance measures such as the SU interruption probability and the mean SU packet delay, and subsequently investigate the influence of the SU sensing rate on these performance measures.

The rest of the paper is structured as follows. Section II presents the system model. In Section III, we provide the performance analysis followed by a discussion of numerical results in Section IV. In Section V we conclude.

\section{SySTEM MOdEL}

We consider a system with $N$ channels used by both PUs and SUs. PUs arrive according to a Poisson process with rate $\lambda_{1}$. Upon arrival, a PU is assigned to any idle channel randomly. A channel occupied by a SU is considered idle for PUs, i.e. the presence of SUs is not detected by PUs. PUs are blocked only when all the channels are occupied by other PUs. The channel holding time of PUs is the same for all channels and has an exponential distribution with rate $\mu_{1}$. Likewise, SUs arrive according to a Poisson process with rate $\lambda_{2}$. Upon arrival, a SU enters into a sensing state where a SU detects idle channels if any. The sensing time has an exponential distribution with rate $\sigma$. It is assumed that a SU can detect all idle channels not occupied by any other SU or PU. Also, it is considered that several SUs can sense the channels simultaneously and no collisions between arriving SUs can happen while trying to transmit as in [5]. After finishing sensing, a SU enters the transmission state. The transmission time of SUs has an exponential distribution with rate $\mu_{2}$ for all channels. During this state, a SU can get interrupted by the arrival of a PU that can occupy the channel used by this SU. The SU is then transferred into the sensing state again if the system is not yet full, i.e. if the total number of PUs and other SUs already in the system is less than $N$. Otherwise, the $\mathrm{SU}$ will be discarded. Thus, the SU may switch between these two states several times before exiting the system. Also, arriving SUs are blocked when the total number of SUs (sensing and transmitting) and PUs in the system equals $N$.
To investigate this system, a CTMC with state space $S$ is developed. The system state is denoted by $x=\left(x_{1}, x_{2}, x_{3}\right)$, where $x_{1}, x_{2}$ and $x_{3}$ are the number of PUs, transmitting SUs and sensing SUs in the system respectively. The state space $S$ contains all states such that the sum

$$
s(x)=\sum_{i=1}^{3} x_{i}
$$

is less than or equal to $N$, so that $S=\left\{x \in \mathbb{N}^{3}: s(x) \leq N\right\}$. The infinitesimal generator $Q=\left[q_{x, y}\right]$ of the system is created, where the transition rate $q_{x, y}$ from one state $x$ into another state $y(x \neq y)$ is given as

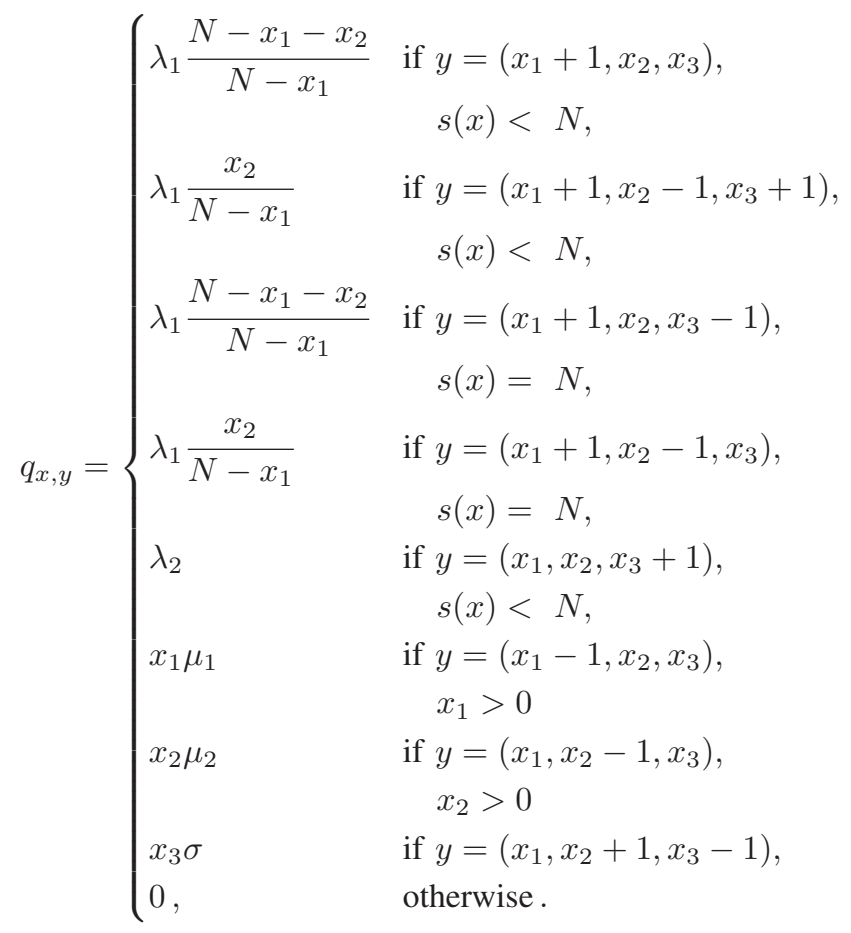

As can be seen, 9 different transition cases are distinguished. The transition rate $\lambda_{1}\left(N-x_{1}-x_{2}\right) /\left(N-x_{1}\right)$ in cases 1 and 3 corresponds to the fraction of $\lambda_{1}$ where an arriving PU doesn't interrupt any transmitting SU, whereas the transition rate $\lambda_{1}\left(x_{2}\right) /\left(N-x_{1}\right)$ in cases 2 and 4 is the fraction of $\lambda_{1}$ where a transmitting SU is interrupted. It is important to note that in cases 1 and 2 no SU is discarded from the system; the interrupted transmitting SU in case 2 is simply transferred to the sensing state. On the other hand, a SU is discarded from the sensing state in case 3 and from the transmitting state in case 4 due to the assumption that $s(x) \leq N$. The transition rate $x_{3} \sigma$ in case 8 is due to the assumption of no channel collisions between simultaneously sensing SUs. The other cases are selfexplanatory. The vector $\pi$ with steady-state probabilities of the CTMC can be computed as the solution of $\pi Q=0$ using the normalization condition $\sum_{x \in S} \pi_{x}=1$, where $\pi_{x}$ denotes the probability for the system to be in state $x$. 


\section{PERformance MEASURES}

Based on the obtained steady-state vector $\pi$, we now derive several performance measures of the system. First, the expected number of transmitting SUs $E\left[\right.$ UTransmit $\left._{\mathrm{su}}\right]$ and sensing SUs $E\left[\mathrm{USense}_{\mathrm{su}}\right]$ are respectively

$$
\begin{gathered}
E\left[\text { UTransmit }_{\mathrm{su}}\right]=\sum_{x \in S} x_{2} \pi_{x}, \\
E\left[\text { USense }_{\mathrm{su}}\right]=\sum_{x \in S} x_{3} \pi_{x} .
\end{gathered}
$$

The SU blocking probability $\gamma$ is the probability that an arriving SU is blocked because of a full system and is given as

$$
\gamma=\sum_{x \in S^{*}} \pi_{x}
$$

where $S^{*} \subset S$ is the set that contains all states satisfying the condition $s(x)=N$.

Based on Little's law, the expected delay of a SU $E\left[D_{s u}\right]$ is then calculated as

$$
E\left[D_{s u}\right]=\left(E\left[\text { USense }_{\mathrm{su}}\right]+E\left[\text { UTransmit }_{\mathrm{su}}\right]\right) /\left(\lambda_{2}-\nu\right),
$$

where $\nu=\lambda_{2} \gamma$ is the fraction of arriving SUs who find a fully occupied system.

Secondly, we are also interested in the SU interruption probability $\alpha$ and the SU discard probability $\beta$. The SU interruption probability $\alpha$ is computed as follows:

$$
\alpha=\sum_{x \in S^{* *}} \pi_{x} \frac{\lambda_{1} x_{2} /\left(N-x_{1}\right)}{D(x)},
$$

where

$$
D(x)= \begin{cases}x_{1} \mu_{1}+x_{2} \mu_{2}+x_{3} \sigma+\lambda_{1}+\lambda_{2} & \text { if } s(x)<N, \\ x_{1} \mu_{1}+x_{2} \mu_{2}+x_{3} \sigma+\lambda_{1} & \text { if } s(x)=N\end{cases}
$$

and $S^{* *}=S \backslash\{(N, 0,0)\}$ denotes the whole state space except the state where $x_{1}=N$ (i.e. the state where the system is fully occupied by PUs and hence there are no SUs in the system).

The SU discard probability $\beta$ is the probability of a SU getting discarded after having entered the system because of lack of space. This probability is given by

$$
\beta=\sum_{x \in S^{* * *}} \pi_{x} \frac{\lambda_{1}}{x_{1} \mu_{1}+x_{2} \mu_{2}+x_{3} \sigma+\lambda_{1}},
$$

where $S^{* * *}=S^{*} \backslash\{(N, 0,0)\}$.

\section{DisCussion OF NuMERICAL RESUlts}

To study the impact of the sensing rate of the SUs, we consider a scenario with $N=20$. The average channel holding time $1 / \mu_{1}$ for PUs is chosen to be the same as the average transmission time $1 / \mu_{2}$ for SUs and equal to $10 \mathrm{~ms}$. We define the offered load of PUs as $\rho_{\text {pu }}=\lambda_{1} /\left(N \mu_{1}\right)$. Since in practice CRNs operate under light PU load, we consider in our examples the case where $\rho_{\text {pu }}=0.3$.

The mean numbers of sensing SUs and transmitting SUs as a function of the sensing rate $\sigma$ are shown in Fig. 1 and Fig. 2

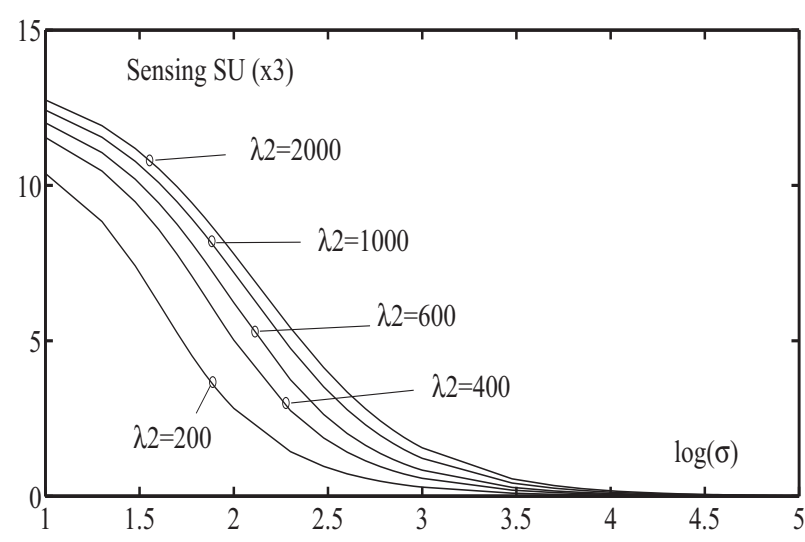

Fig. 1. Average number of sensing SUs $E\left[\right.$ USense $\left._{\mathrm{su}}\right]$ versus sensing rate $\sigma$ in $\log _{10}$ scale for various $\lambda_{2}=200,400,600,1000,2000, \rho_{\mathrm{pu}}=0.3$, $N=20$ and $1 / \mu_{1}=1 / \mu_{2}=10 \mathrm{~ms}$.

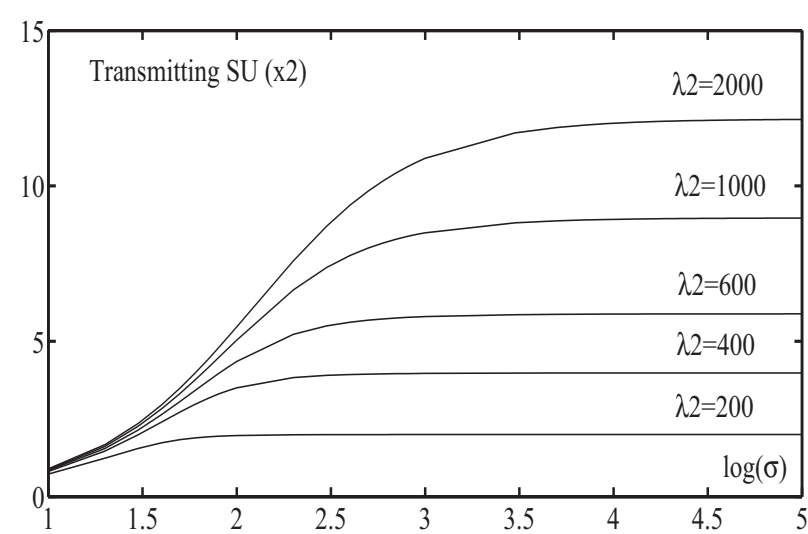

Fig. 2. Average number of transmitting SUs $E$ [UTransmit $\left.\mathrm{U}_{\mathrm{su}}\right]$ versus sensing rate $\sigma$ in $\log _{10}$ scale for various $\lambda_{2}=200,400,600,1000,2000, \rho_{\mathrm{pu}}=0.3$, $N=20$ and $1 / \mu_{1}=1 / \mu_{2}=10 \mathrm{~ms}$.

respectively. We observe that an increase of the sensing rate $\sigma$ transfers more SUs from the sensing state into the transmitting state. Eventually, for high values of $\sigma$, all sensing users will be transferred into the transmitting state. This can be seen from the figures where for high $\sigma$, the average number of sensing SUs approaches zero and the average number of transmitting SUs converges to a limiting value. This is possible because there is always an available server for a sensing SU to start transmitting.

Fig. 3 shows the mean delay of SUs as a function of the sensing rate $\sigma$ for different SU arrival rates $\lambda_{2}$. As can be seen, the sensing rate has a clear impact on the delay of SUs when $\sigma$ is low. The mean delay of a SU will tend to reach a fixed value for higher values of $\sigma$. This corresponds to the case when most of the SUs are in the transmission state and consequently increasing $\sigma$ will not affect the mean delay of SUs.

The SU interruption probability $\alpha$ as a function of $\sigma$ is given 


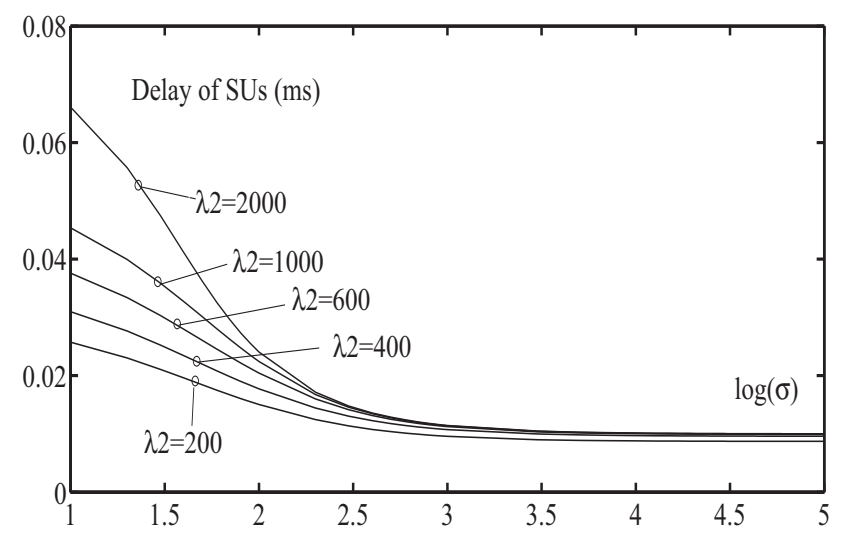

Fig. 3. Average delay of SUs $E\left[D_{s u}\right]$ versus sensing rate $\sigma$ in $\log _{10}$ scale for various $\lambda_{2}=200,400,600,1000,2000, \rho_{\mathrm{pu}}=0.3, N=20$ and $1 / \mu_{1}=$ $1 / \mu_{2}=10 \mathrm{~ms}$

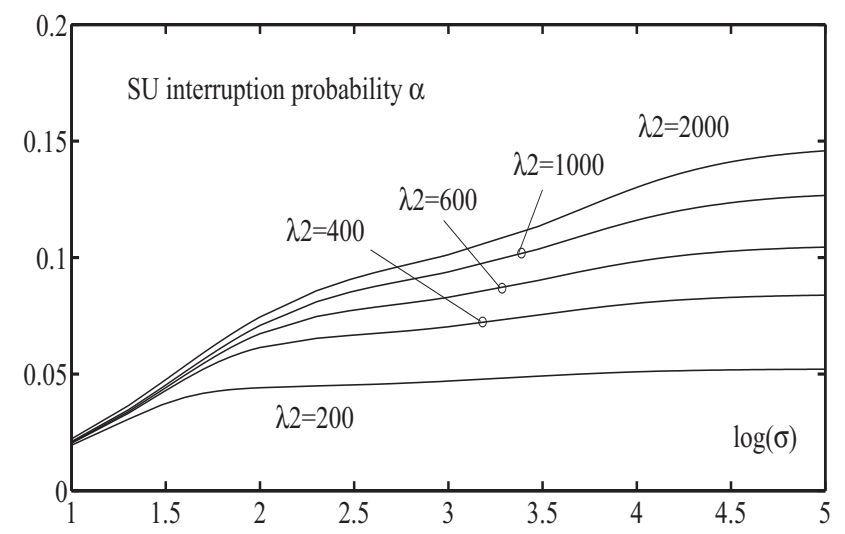

Fig. 4. SU interruption probability $\alpha$ versus sensing rate $\sigma$ in $\log _{10}$ scale for various $\lambda_{2}=200,400,600,1000,2000, \rho_{\mathrm{pu}}=0.3, N=20$ and $1 / \mu_{1}=$ $1 / \mu_{2}=10 \mathrm{~ms}$.

in Fig. 4. As $\sigma$ increases, the probability of SU interruption by an arriving PU increases. This is explained by the fact that an increase of $\sigma$ will transfer more SUs into the transmitting state and consequently an arriving PU will have more chances to interrupt a SU. Also, comparing Fig. 3 and Fig. 4, we can see that the increased interruption probability does not have impact on the delay experienced by SUs, but signifies the increased rate at which SUs might have to switch to different channels It is interesting to note that even when the SU arrival rate $\lambda_{2}$ is high $\left(\lambda_{2}=2000\right)$, the probability of SU interruption by an arriving PU is low $(<0.15)$ for all values of $\sigma$ investigated.

The SU blocking probability $\gamma$ is given in Fig. 5. For increasing $\sigma$, the blocking probability of SUs decreases. This can be explained by a decrease of the total time a SU spends in the system, which creates more room for an arriving SU to enter the system.

Fig. 6 shows the SU discard probability $\beta$ versus $\sigma$. For increasing $\sigma$ (up to $\log _{10} \sigma=3$ ), the SU discard probability

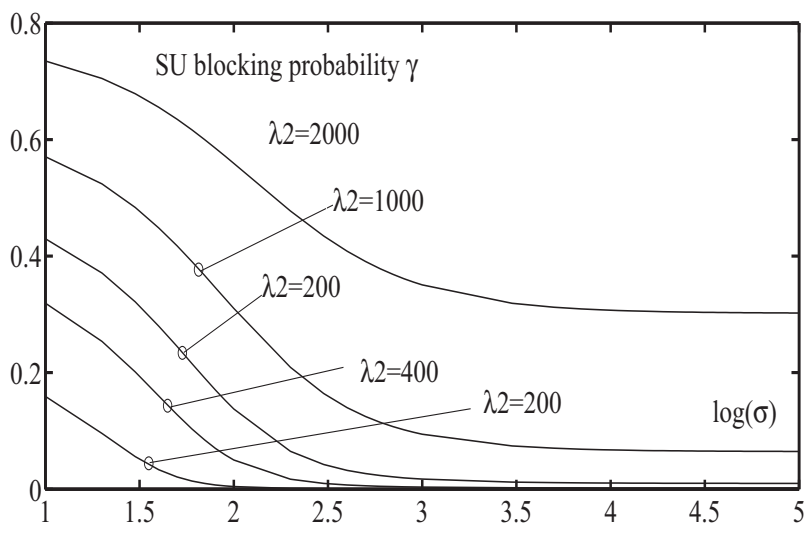

Fig. 5. SU blocking probability $\gamma$ versus sensing rate $\sigma$ in $\log _{10}$ scale for various $\lambda_{2}=200,400,600,1000,2000, \rho_{\mathrm{pu}}=0.3, N=20$ and $1 / \mu_{1}=$ $1 / \mu_{2}=10 \mathrm{~ms}$.

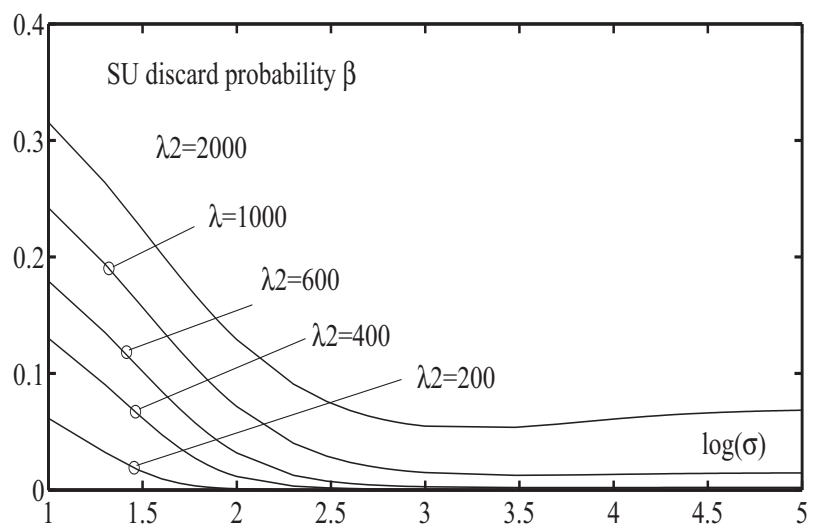

Fig. 6. SU discard probability $\beta$ versus sensing rate $\sigma$ in $\log _{10}$ scale for various $\lambda_{2}=200,400,600,1000,2000, \rho_{\mathrm{pu}}=0.3, N=20$ and $1 / \mu_{1}=$ $1 / \mu_{2}=10 \mathrm{~ms}$

will decrease. This is also due to a decrease of the time a SU spends in the system, which in turn leads to less possibilities to discard the SU. It has been noticed that a further increase of $\sigma$ will slightly increase the probability $\beta$ for a high SU arrival rate $\lambda_{2}=2000$. This phenomenon is due to the fact that, when increasing the sensing rate, SUs depart from the system earlier and fewer new SUs are blocked. The reduced blocking probability increases the SU load admitted to the system and, beyond some point, leads to a slightly increased discard probability.

It is important to note that the system has also been investigated analytically for other values of $N$ between 5 and 30 , and for various service rates of SUs and PUs including cases where $\mu_{1} \neq \mu_{2}$ and the qualitative behavior of the above considered performance characteristics has not changed. By careful investigation of Figs. 3, 6 and 5, we can see a region of $3<\log _{10} \sigma<3.5$ that seems to optimize the operation of SUs for the given parameter set. Indeed, in this region, 
the mean SU delay, the SU discard probability and the SU blocking probability approach minimum values. There is no point to further increase $\sigma$ since the interruption probability will then increase as well.

\section{CONCLUSION}

In this paper, we have analyzed the impact of the SU sensing rate $\sigma$ on some performance measures of SUs in a CRN through a finite CTMC. It has been shown that $\sigma$ has a huge impact on the delay of SUs in CRNs. Also for a given parameter set, there seems to be a region of $\sigma$ that optimizes SU operation. Finding such a region is important because, from a practical point of view, the earlier this region starts, the more chance a $\mathrm{SU}$ is able to find idle channels.

\section{ACKNOWLEDGEMENT}

The second author is a Postdoctoral Fellow with the Research Foundation, Flanders (FWO-Vlaanderen), Belgium. This research has been funded by the Interuniversity Attraction Poles Programme initiated by the Belgian Science Policy Office.

\section{REFERENCES}

[1] J. Mitola, "Cognitive radio: an integrated agent architecture for software defined radio," PhD Dissertation, KTH, Stockholm, Sweden, Dec. 2000

[2] B.A. Fette (ed.), "Cognitive radio technology," 2006, Elsevier.

[3] I.F. Akyildiz, W.-Y. Lee, M.C. Vuran, S. Mohanty, "A survey of spectrum management in cognitive radio networks," IEEE Communications Magazine, April 2008, pp. 40-48.

[4] Y. Huang and J. Wang, "A multi-class preemptive priority cognitive radio system with random interruption discipline," in Proceedings of the 5th International Conference on Queueing Theory and Network Applications, 2010, pp. 162-168.

[5] Beibei Wang, Zhu Ji, K.J.R. Liu and T.C. Clancy, "Primary-prioritized Markov approach for dynamic spectrum allocation," IEEE Trans. Wireless Commun., vol. 8, no. 4, pp. 1854-1865, 2009.

[6] E. Wong and C. Foh, "Analysis of cognitive radio spectrum access with finite user population," Communications Letters, IEEE, vol. 13, no. 5, pp. 294-296, May 2009.

[7] A. S. Qing Zhao, Lang Tong and Y. Chen, "Decentralized cognitive MAC for opportunistic spectrum access in ad hoc networks: A pomdp framework," IEEE J. Sel. Areas Commun., vol. 25, no. 3, pp. 589-600, 2007.

[8] L. T. Qianchuam Zhao, Stefan Gierhofer and B. Sadler, "Opportunistic spectrum access via periodic channel sensing," Signal Processing, IEEE Transactions on, vol. 56, no. 2, pp. 785-796, 2008.

[9] X. G. Xin Li, Qianchuam Zhao and L. Tong, "Optimal cognitive access of Markovian channels under tight collision contraints," IEEE J. Sel. Areas Commun., vol. 29, no. 4, pp. 746-756, 2011.

[10] L. Wang, C. Wang, and C. Chang, "Modeling and analysis for spectrum handoffs in cognitive radio networks," Mobile Computing, IEEE Transactions on, vol. 11, no. 9, pp.1999-1513, 2011.

[11] C. Zhang, X. Wang, and J. Li, "Cooperative cognitive radio with priority queueing analysis," in Proceedings of the 2009 IEEE International Conference on Communications, ser. ICC'09. Piscataway, NJ, USA: IEEE Press, 2009, pp. 4672-4676. [Online]. Available: http://dl.acm.org/citation.cfm?id=1817770.1818142

[12] H.-P. Shiang and M. van der Schaar, "Queuing-based dynamic channel selection for heterogeneous multimedia applications over cognitive radio networks," Trans. Multi., vol. 10, no. 5, pp. 896-909, Aug. 2008. [Online]. Available: http://dx.doi.org/10.1109/TMM.2008.922851

[13] Y. Song and J. Xie, "Performance analysis of spectrum handoff for cognitive radio ad hoc networks without common control channel under homogeneous primary traffic," in INFOCOM, 2011 Proceedings IEEE, April 2011, pp. 3011-3019.
[14] C.-W. Wang, L.-C. Wang, and F. Adachi, "Modeling and analysis for reactive-decision spectrum handoff in cognitive radio networks," in Global Telecommunications Conference (GLOBECOM 2010), 2010 IEEE, Dec. 2010, pp. 1-6.

[15] A. Laourine, S. Chen, and L. Tong, "Queuing analysis in multichannel cognitive spectrum access: A large deviation approach," in INFOCOM 2010 Proceedings IEEE, March 2010, pp. 1-9. 